\title{
Electron Holography of Naturally Occurring Nanomagnets in Rocks
}

\author{
R.E. Dunin-Borkowski, ${ }^{*}$ R.J. Harrison, ${ }^{* *}$ and A. Putnis*** \\ * Department of Materials Science and Metallurgy, University of Cambridge, Pembroke Street, \\ Cambridge CB2 3QZ, United Kingdom \\ ** Department of Earth Sciences, University of Cambridge, Downing Street, Cambridge CB2 \\ 3EQ, United Kingdom \\ *** Institut für Mineralogie, Corrensstrasse 24, D-48149 Münster, Germany
}

Small particles of magnetite $\left(\mathrm{Fe}_{3} \mathrm{O}_{4}\right)$, which are formed by solid-state processes such as subsolvus exsolution, are the dominant carriers of strong and stable remanent magnetization in rocks. Here, we use off-axis electron holography in the transmission electron microscope (TEM) to image the magnetic remanent states of a natural, finely-exsolved intergrowth of submicron magnetite blocks in an ulvöspinel $\left(\mathrm{Fe}_{2} \mathrm{TiO}_{4}\right)$ matrix. The observations described below were carried out at $300 \mathrm{kV}$ using a Philips CM300-ST TEM equipped with a field-emission source, a Lorentz lens, an electrostatic biprism and a Gatan Imaging Filter.

Figures $1 \mathrm{a}$ and $\mathrm{b}$ show Fe and Ti chemical maps of a representative area of the sample.

Ulvöspinel-rich exsolution lamellae subdivide the original titanomagnetite grain into an array of magnetite-rich blocks, which are roughly equidimensional. Figure 2 shows the magnetic microstructure of the same region measured using electron holography [1]. Each image corresponds to a different magnetic remanent state, acquired with the sample in field-free conditions. The outlines of the magnetite-rich regions are marked in white, while the direction of the local magnetic induction is indicated using arrows. The spacing of the black contours is inversely proportional to the strength of the magnetic induction in the plane of the sample. Single-domain and vortex states in individual blocks, as well as magnetostatic interaction fields between them, are imaged at a spatial resolution approaching the nanometer scale. The images reveal an extremely complicated magnetic microstructure, in which both the shapes of the blocks and magnetostatic interactions between them are responsible for the stability of the remanent magnetization. Magnetic superstates, in which clusters of blocks act collectively to form vortex and multi-domain states that have zero net magnetization, are also observed in Fig. 2. Figure 3a shows a remanent hysteresis loop, measured from images similar to those in Fig. 2. The graph tends to a value below unity because the magnetic microstructure is never saturated in the applied field direction in the remanent state. Figure $3 \mathrm{~b}$ shows the magnetic microstructure of a small magnetite-rich region that is magnetically antiparallel to its neighbors. The magnetization direction of the small block follows the flux return paths of the larger adjacent blocks. This observation may provide insight into one of the causes of self-reversed thermo-remanent magnetization, in which the magnetization directions of some blocks can point antiparallel to the applied field due to strong magnetostatic interactions with their neighbors [2].

\section{References}

[1] R.J. Harrison et al., Proc. Nat. Acad. Sci. 99 (2002) 16556.

[2] This research was supported by the Royal Society, the Engineering and Physical Sciences Research Council, and the Deutsche Forschungsgemeinschaft. Discussions with Prof.

M. R. Scheinfein are gratefully acknowledged. 

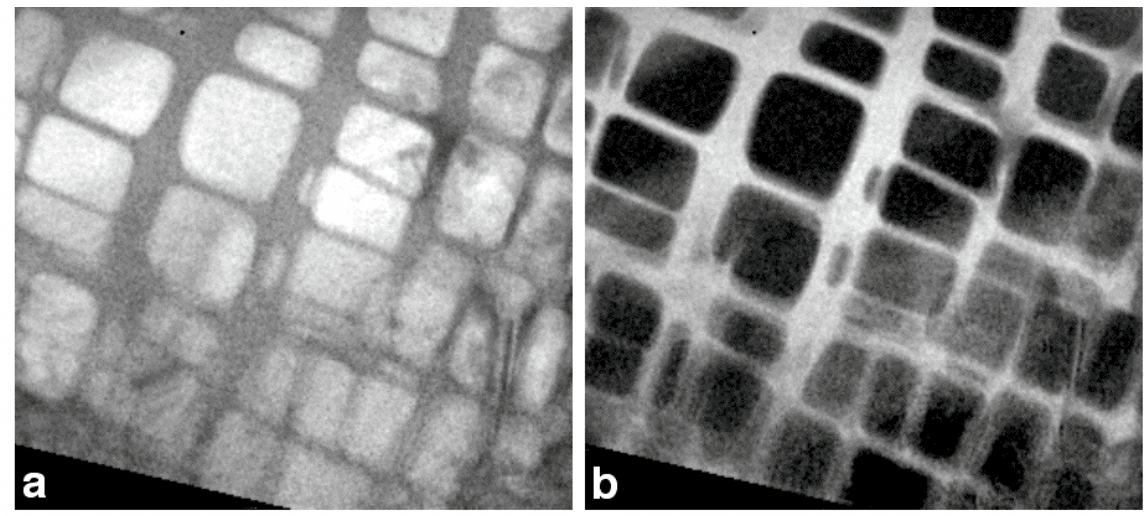

$200 \mathrm{~nm}$

FIG. 1. Three-window background-subtracted elemental maps acquired from a naturally occurring titanomagnetite sample using a Gatan Imaging Filter using a) the Fe L edge, and b) the Ti L edge.

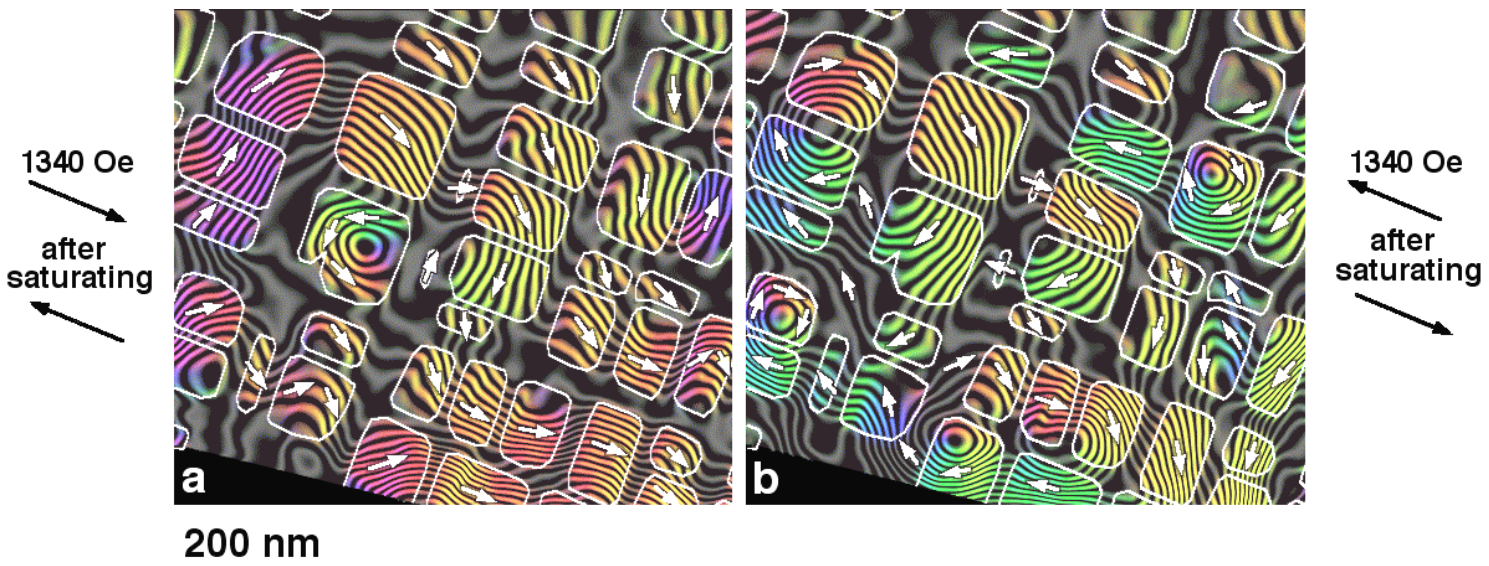

FIG. 2. Two magnetic remanent states of the region shown in Fig. 1 measured using electron holography. a) was obtained after saturating the sample toward the top left, then applying an in-plane field of 1340 Oe toward the bottom right, after which the external magnetic field was removed. b) was obtained after applying identical fields in the opposite direction.
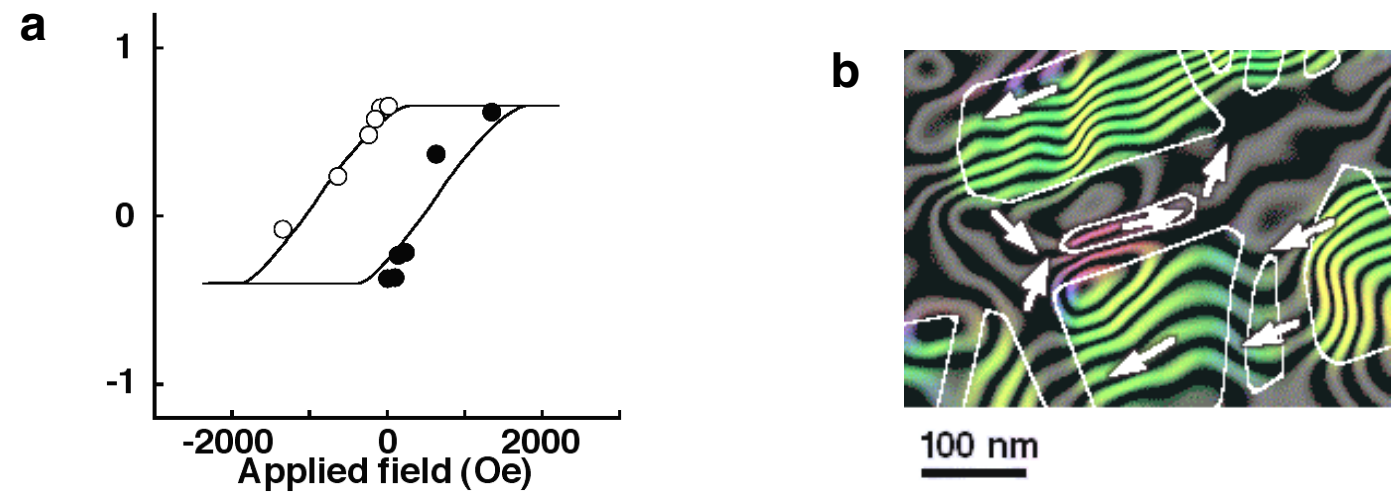

$100 \mathrm{~nm}$

FIG. 3. a) Remanent hysteresis loop obtained from images similar to those shown in Fig. 2 by plotting the average fraction of the magnetic induction in the magnetite-rich blocks in the direction of the applied field. b) Magnetic microstructure of a small magnetite-rich region that is magnetically antiparallel to its larger neighbors. 